\title{
大气主要污染物与细菌气溶胶在冰核核化过程 中的作用：对液滴冻结温度的影响
}

\author{
王亚玲，杜点*，梁宗敏，李梓铭 \\ 中国科学院研究生院, 北京 100049 \\ * 联系人, E-mail: ruidu2000@yahoo.com \\ 收稿日期: 2011-12-28; 接受日期: 2012-02-24 \\ 国家自然科学基金(批准号: 40875081, 41175135)资助
}

摘要冰核细菌气溶胶作为有效云凝结核与冰核可能在大气物理与化学过程和气象过程 中起重要作用, 其核化特性也受大气和云中气溶胶的物理、化学性质的影响. 本研究依据 Vali 均匀液滴冻结实验的原理, 采用改进的液滴冻结仪分别测试了大气典型污染物(硫酸铵; 一元羧酸(MCA): 甲酸和乙酸钠; 二元羧酸(DCA): 乙二酸和丙二酸)与不同浓度的细菌菌液 (冰核活性细菌Pseudominas syringae pv. lachrymans(PS))和非冰核细菌P. syringae pv. panici $\left.\left(\mathrm{PS}^{0}\right)\right)$ 的冻结温度. 结果显示: 不同浓度的 $\mathrm{MCA} / \mathrm{DCA}\left(50,75,100 \mu \mathrm{mol} \mathrm{L} \mathrm{L}^{-1}\right)$ 和硫酸铵 $(100$, $\left.200,300,400 \mu \mathrm{mol} \mathrm{L}{ }^{-1}\right)$ 溶液液滴 $(10 \mu \mathrm{L})$ 的平均冻结温度范围在 $-17.0 \sim-20.0^{\circ} \mathrm{C}$, 相比超纯水液 滴的冻结温度 $(-20.6 \pm 1.6)^{\circ} \mathrm{C}$, 典型大气污染物的加入并未显著提升超纯水液滴的冻结温度, 而且污染物溶液浓度的改变对冻结温度没有表现出明显的规律性. 冰核细菌 PS 和非冰核细 菌菌液 $\mathrm{PS}^{0}$ 与大气主要污染物( 乙酸钠、乙二酸和硫酸铵)混合液的液滴冻结温度测试结果发 现: 这些污染物质不同程度降低了细菌 PS 与 $\mathrm{PS}^{0}$ 菌液的冻结温度, 即使 PS 在较低浓度下也 可促进大气主要污染物乙酸钠的冻结核活性, 提高其冻结温度; 当浓度增至 $10^{6}$ cells $\mathrm{mL}^{-1}$ 时, 则能显著改变污染物质的冻结核活性; 但未发现 $\mathrm{PS}^{0}$ 有此特性. 复合污染物溶液浓度接近当 前污染大气水平时, 对冰核细菌 PS 和较高浓度级数 $\left(10^{6}\right.$ cells $\left.\mathrm{mL}^{-1}\right)$ 的 $\mathrm{PS}^{0}$ 的冻结核活性都显 示出了促进效应, 表明在当前大气环境条件下, 细菌气溶胶确实有可能影响降水过程和气候 变化. 冰核活性细菌与非活性冰核细菌混合后, 只有当冰核细菌浓度级数达到 $10^{6} \mathrm{cells} \mathrm{mL}^{-1}$ 时, 在混合液中才可表现出高效冰核活性. 冰核细菌的浓度值是影响其活性的重要因素, 使 其在溶液中显示高效冰核活性的菌浓度阈值级数是 $10^{6}$ cells $\mathrm{mL}^{-1}$; 本研究结果对于人工影响 天气过程中, 人工冰核的选择和配比方法优化方面具有一定的科学意义.

\section{关键词 \\ 冰核 \\ 细菌气溶胶 \\ 冰核活性细菌 \\ 大气主要污染物 \\ 核化 \\ 冻结温度}

云作为产生降水的母体和影响地球能量辐射传 输的重要影响因素, 其在全球气候变化的重要作用
已经受到广泛重视. 模式研究表明: 冰核数量能直 接影响气候变化, 冰核浓度增加一个数量级, 全球云

英文引用格式: Wang Y L, Du R, Liang Z M, et al. The interaction between atmospheric typical pollutants and bacteria aerosols in ice heterogeneous nucleation: Effect of droplets freezing temperature (in Chinese). Sci Sin Terrae, 2012, 42: 692-700 
的净辐射强迫便增加 $1 \mathrm{~W} \mathrm{~m}^{-2[1]}$. 生物气溶胶(如细 菌、真菌和藻类)可作为云凝结核(Cloud condensation nuclei, CCN)和冰核(Ice nuclei, IN), 改变大气云的化 学和物理过程, 从而影响大气降水、大气化学和微生 物地球化学循环过程 ${ }^{[2,3]}$. 细菌气溶胶作为极具活性 的一类生物气溶胶, 除了其本身异质核化能力外, 对 其他气溶胶粒子的化学成分和物理特性的影响已成 为生物气溶胶的气候效应的重要研究方向. Morris 等 ${ }^{[4]}$ 总结了 Pseudomonas syringae 等冰核细菌在大气 降水过程中可能具有的重要作用.

冰核细菌在大气中附着于其他颗粒而存在于气 溶胶小系统中, 其生物活性与气溶胶其他成分密切 相关. 已有的研究显示有机气溶胶占大气气溶胶质 量浓度的 20\% 90\% ${ }^{[5]}$; 已经成为全球云凝结核和冰 核的重要组成成分 ${ }^{6]}$. 大气中最频繁出现的有机颗粒 物主要是低分子量的单元羧酸 MCA(mono-carboxylic acids，甲酸、乙酸、乳酸、丙酸等) 和二元羧酸 DCA (dicarboxylic acids, 乙二酸、丁二酸、顺丁烯二酸、戊 二酸等), 而且单元羧酸占有机羧酸质量浓度的 $71 \%$, 二元羧酸占大约 $29 \%^{[7]}$; 气溶胶中甲酸盐和乙酸盐的 浓度分别在 $0.02 \sim 5.3$ 和 $0.03 \sim 12.4 \mathrm{nmol} \mathrm{m}^{-3}$, 约 $34 \%$ 77\% 的甲酸盐和 21 66\%的乙酸盐存在于气溶 胶细粒子中 ${ }^{8]}$. 气溶胶中的 DCA 主要是乙二酸 $(\mathrm{C} 2)$, 其次的是丙二酸 $\mathrm{C} 3$ 和丁二酸 $\mathrm{C} 4^{[9,10]}$. 硫酸盐(如 $\left.\left(\mathrm{NH}_{4}\right)_{2} \mathrm{SO}_{4}\right)$ 被认为是 $\mathrm{CCN}$ 的最主要的成分 ${ }^{[1]}$. 室内 模拟实验已经证实, 空气微生物可以利用大气中 DCA 进行生长代谢, 从而改变和影响大气化学特 性 ${ }^{[12]}$. 目前关于大气典型污染物以及冰核细菌的异 质核化特性的研究较多地集中在其核化能力与物化 和生化特性的低温测试方面, 这需要灵敏可控的温 湿条件以及晶体显微技术 ${ }^{[13]}$, 受高精的测试设备和 交叉学科知识的限制, 目前还有许多关于有机气溶 胶粒子作为大气云凝结核和冰核的成核活性的科学 问题尚未找到答案, 其中有关主要大气污染物与重 要的冰核细菌的交互作用对核化活性与过程可能产 生的影响的研究开展的更为有限, 本研究以典型冰 核细菌 P. syringae pv. lachrymans(PS)作为研究对象, 利用室内模拟试验测定大气气溶胶的主要污染成分 对其冻结温度的影响, 探求其可能在大气冰相核化 过程中的作用效应.

\section{1 材料和方法}

自 Maki 和 Willoughby ${ }^{[14]} 1974$ 年分离到冰核活性 细菌 P. syringae 以来, 目前国际公认 P. syringae 是分 布最广和活性最强的冰核细菌模式菌种 ${ }^{[15]}$.

外场试验观测发现 2002 2003 年期间, 北京市内 大气细颗粒物 $\mathrm{PM}_{2.5}$ 的二元羧酸中乙二酸平均浓度范 围在 1.2 4.6 $\mu \mathrm{mol} \mathrm{L} \mathrm{L}^{-1}$ 附近波动; 丙二酸和丁二酸的 季节平均浓度范围是 $0.2 \sim 0.6 \mu \mathrm{mol} \mathrm{L} \mathrm{L}^{-1}$, 戊二酸浓度

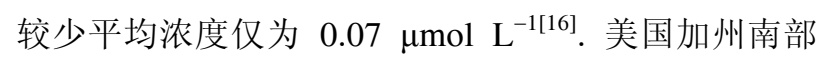
和东京降水中二元羧酸的浓度分别是 $0.9 \sim 8.7$ 和 $0.1 \sim$ $4.6 \mu \mathrm{mol} \mathrm{L}{ }^{-1[17,18]}$. 北京降水中主要的水溶性有机酸 是甲酸、乙酸和草酸, 占阴离子总浓度的 $2 \%{ }^{[19]}$. 因 此我们选甲酸、乙酸盐、乙二酸(草酸)和丙二酸代表 大气主要有机污染物模拟冰核细菌在云中可能存在 的微化学环境.

\section{1 实验菌种}

实验采用由中国科学院微生物研究所中国普通 微生物保藏中心提供的丁香假单胞菌㯟离致病变种 (Pseudomonas syringae pv. panici)和中国农业科学院 植物保护研究所植物病虫害生物学国家重点实验室 所提供的丁香假单胞菌黄瓜致病变种 $(P$. syringae pv. lachrymans)冻干管保存的纯种菌, 后者为植保所植 物病虫害生物学国家重点实验室所篮选出的我国冰 核活性最强的冰核细菌 P. syringae 的变种代表菌株, 为下文讨论方便, 分别依据其拉丁学名首字母缩写, 将前者无冰核活性的丁香假单胞菌㯟离致病变种编 码为 $\left(\mathrm{PS}^{0}\right)$ 作为大气中普遍存在的普通细菌的代表, 而将有冰核活性的丁香假单胞菌黄瓜致病变种编码 为 $(\mathrm{PS})$ 作为冰核细菌的代表.

\section{2 菌种的活化}

将冻干管保存菌种用适量无菌水混匀, 用滴管 吸出转移至试管斜面 NA 培养基中, $30^{\circ} \mathrm{C}$ 培养 $24 \mathrm{~h}$, 经多次转接活化后, 在 NA 培养血上培养纯化分离, 再转接试管斜面培养基作为常用菌种 $4^{\circ} \mathrm{C}$ 保存. 实验 用营养琼脂 NA-CM107 培养基，由北京陆桥技术有 限公司生产. 


\section{3 测试菌液及大气典型污染物混合液的配制}

\subsection{1 菌液}

将接种培养 $\left(\right.$ 室温 $<25^{\circ} \mathrm{C}$ ) 并在 $4^{\circ} \mathrm{C}$ 冰箱冷藏保存至 少一周以上的细菌培养血取出, 在无菌操作台用无 菌菌环刮取培养基上的菌落, 移到无菌超纯水中, 震 荡摇匀, 根据前期测定好的 $\mathrm{OD}_{600}$ 值与细菌平板计数 关系式, 按 $\mathrm{OD}_{600}$ 值配制所需浓度的菌悬液. 而浓度 较低的菌悬液, 则通过梯度稀释法将已知浓度的母 菌悬液稀释而成. 大气水中总细菌浓度数量级约 $10^{3} \sim 10^{5}$ cells $\mathrm{mL}^{-1[20]}$, 因此本研究的配制菌液浓度范 围是 $10^{3} \sim 10^{6}$ cells $\mathrm{mL}^{-1}$.

\subsection{2 污染物与冰核活性细菌 PS、普通细菌 PS $^{\mathbf{0}}$ 的混合液}

大气典型污染物溶液: 用美国 Milipore 公司生产 的离子塞柱纯净水过滤仪过滤产生的 $18 \Omega$ 超纯水配 制, 甲酸、乙酸钠、乙二酸和丙二酸溶液各 3 个浓度 $\left(50,75,100 \mu \mathrm{mol} \mathrm{L}^{-1}\right)$, 硫酸铵溶液 4 个浓度 $(100,200$, $\left.300,400 \mu \mathrm{mol} \mathrm{L}^{-1}\right)$. 将乙酸钠、乙二酸和硫酸铵三种 污染物按不同浓度配比制成复合溶液 P1 $(100 \mu \mathrm{mol}$ $\left.\mathrm{L}^{-1}: 100 \mu \mathrm{mol} \mathrm{L} \mathrm{L}^{-1}: 200 \mu \mathrm{mol} \mathrm{L}\right), \mathrm{P} 2\left(50 \mu \mathrm{mol} \mathrm{L}^{-1}: 50\right.$ $\left.\mu \mathrm{mol} \mathrm{L} \mathrm{L}^{-1}: 100 \mu \mathrm{mol} \mathrm{L} \mathrm{L}^{-1}\right)$ 和 P3 $\left(25 \mu \mathrm{mol} \mathrm{L} \mathrm{L}^{-1}: 25 \mu \mathrm{mol}\right.$ $\left.\mathrm{L}^{-1}: 50 \mu \mathrm{mol} \mathrm{L}{ }^{-1}\right)$.

污染物与菌液的混合液: 将单种污染物 (乙酸 钠、乙二酸和硫酸铵 $)$ 分别与不同浓度级数 $\left(10^{4}, 10^{6}\right.$ cells $\mathrm{mL}^{-1}$ )的 PS 和 $\mathrm{PS}^{0}$ 配制混合液; 另将污染物复 合溶液 $(\mathrm{P} 1, \mathrm{P} 2, \mathrm{P} 3)$ 与不同浓度级数 $\left(10^{3}, 10^{4}, 10^{5}, 10^{6}\right.$ cells $\left.\mathrm{mL}^{-1}\right)$ 的 PS 和 $\mathrm{PS}^{0}$ 交叉配制成待测混合液.

\subsection{3 冰核细菌 PS 与普通细菌 $\mathrm{PS}^{0}$ 的混合菌液}

将 $10^{6}$ cells $\mathrm{mL}^{-1}$ 的 PS 菌液和 $\mathrm{PS}^{0}$ 菌液, 按体积 比 $2: 1,1: 1,1: 2$ 混合调制成 3 个混合菌液 A1, A2, A3, 同样方法配制 $10^{5}$ 和 $10^{4}$ cells $\mathrm{mL}^{-1}$ 混合菌液 B1, B2, $\mathrm{B} 3$ 和 $\mathrm{C} 1, \mathrm{C} 2, \mathrm{C} 3$ 然后分别加入复合污染物溶液, 使 最终混合液中 3 种污染物 (乙酸钠、乙二酸和硫酸铵) 溶质浓度分别是 $50,50,100 \mu \mathrm{mol} \mathrm{L}^{-1}$.

\section{4 溶液液滴冻结温度的测定}

本实验采用中国气象科学研究院杨绍忠和酆大 雄 ${ }^{[21]}$ 所研制的均匀水滴冻结仪, 冻结温度的测试精 度是 $0.2^{\circ} \mathrm{C}$, 仪器冷台的密闭小室尺寸： $140 \mathrm{~mm} \times 140$ $\mathrm{mm} \times 50 \mathrm{~mm}$; 液滴感应件尺寸: $10 \mathrm{~mm} \times 10 \mathrm{~mm}$, 相邻 感应片的中心间距是 $18 \mathrm{~mm}$; 温度调控由 $818 \mathrm{P} 4$ 欧陆 控温仪采用 MCGS(Monitor And Control Generated System)编程控温程序控制仪器进行线性降温, 本实 验以 $2^{\circ} \mathrm{C} \mathrm{min}$ 匀速降温. 液滴滴定前按文献描述的 方法在冷台上均匀分布的 47 个感应位点上滴加憎水 膜, 随后采用大龙医疗设备(上海)有限公司生产的 $5 \sim 50 \mu \mathrm{L}$ 微量大龙移液枪依次将 $10 \mu \mathrm{L}$ 溶液液滴滴在 已形成憎水膜的感应位点上. 由于溶液液滴尺度是 影响核化的一个重要因素, 本研究所采用的液滴大 小, 是基于预研究中自 2.5 20 $\mu \mathrm{L}$ 不同液滴大小的测 试结果(未显示), 同时又参考其他同类液滴冻结实验 研究中所采用的液滴大小而确定的. 从 $0^{\circ} \mathrm{C}$ 开始匀速 降温, 液滴冻结, 脉冲信号被检测器自动记录并输 出检测结果. 如遇降温曲线弯曲或在仪器降温范围 内液滴仍不冻结, 记录并舍弃这些液滴数据, 统计时 调整冻结液滴总数. 为保证数据的统计意义, 上述操 作至少重复五次, 使检测结果有效数据保持在 200 个 以上.

\section{5 液滴中冻结核的累积浓度的计算}

本研究中, 溶液冻结核的累积浓度计算采用如 下方程 ${ }^{[22]}$ :

$$
K(\theta)=\left[\ln \left(N_{0}\right)-\ln N(\theta)\right] / V,
$$

式中 $K(\theta)$ 是待测溶液液滴的冻结核的累积浓度, $N(\theta)$ 是当温度是 $\theta$ 时, 待测液滴中尚未冻结的液滴数 量, $N_{0}$ 则是待测液滴的总数量, $V$ 是单个液滴的体积, 单位是 $\mathrm{cm}^{3}$.

\section{2 结果和讨论}

\section{1 典型大气污染物对超纯水液滴冻结温度的影响}

图 1 是不同浓度的大气典型污染物对超纯水液 滴冻结温度的影响, 实验测试结果显示: 单元羧酸和 二元羧酸以及硫酸铵, $10 \mu \mathrm{L}$ 液滴的冻结温度范围在 $-17.0 \sim-20.0^{\circ} \mathrm{C}$, 空白对照超纯水的平均冻结温度是 $(-20.6 \pm 1.6)^{\circ} \mathrm{C}$. 典型大气污染物的加入并没有明显升 高超纯水液滴冻结温度; 设定浓度范围内, 甲酸、乙 酸钠、乙二酸、丙二酸和硫酸铵污染物质溶液液滴冻 结与浓度变化之间没有规律, 而且相比测量的平均 标准偏差, 不同浓度污染物溶液液滴平均冻结温度 


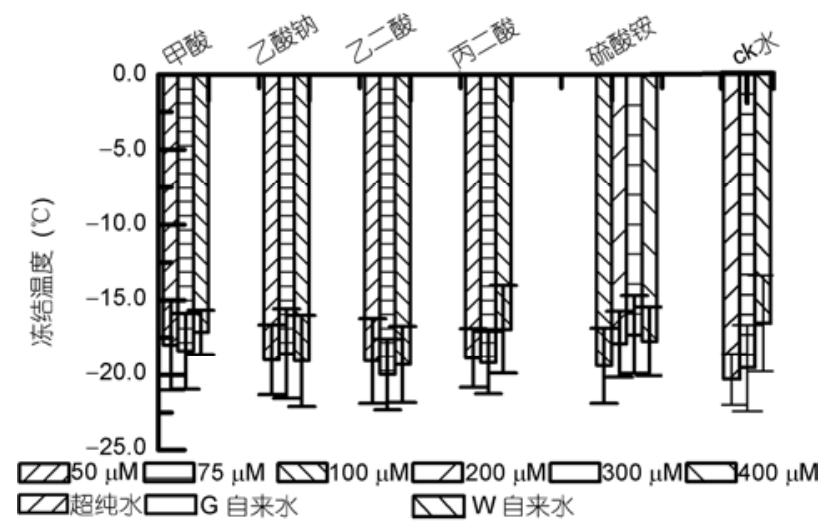

图 1 不同浓度典型大气污染物对超纯水液滴冻结温度的 影响

差异甚至可忽略. 这与 $\mathrm{Du}$ 和 $\mathrm{Ariya}^{[23]}$ 利用加拿大 McGill 大学研制的冻结核计数仪所测得 $5 \mu \mathrm{L}$ 混合态 的二元羧酸(C2-C6, 叠加浓度 50 250 $\mu \mathrm{mol} \mathrm{L}^{-1}$ )平均 冻结温度范围 $(-24.1 \pm 2.8) \sim(-21.3 \pm 3.9)^{\circ} \mathrm{C}$, 相对于超 纯水液滴 $\left((-22.6 \pm 3.5)^{\circ} \mathrm{C}\right)$, 对超纯水冻结温度的促进 作用并不显著的结论一致. 也与 Mortazavi 等 ${ }^{[15]}$ 的结 果一致: 有机分子草酸、丙二酸和丁二酸 $\left(500 \mu \mathrm{mol} \mathrm{L}^{-1}\right)$ 冻结温度范围是 $-17.3 \sim-20.1^{\circ} \mathrm{C}$, 相比超纯水 $(-25.5 \pm$ 2) ${ }^{\circ} \mathrm{C}$ 对冰核化的影响很小, 甚至认为所引起的较少的 温度增高更可能是由溶质中的杂质盐引起的.

Fukuta ${ }^{[24]}$ 发现乙二酸和已二酸在 -10 和 $-9^{\circ} \mathrm{C}$ 都可 以做冰核. 目前关于有机物质产生冰核活性的观点 有: 可能是由极性或高结晶能力特性所诱发; 也可能 由于晶格结构较差其晶体裂缝中的电场诱发了凝华 成核的活性位点, 例如某些氨基酸的核化 ${ }^{[25]}$; 此外 有机化合物也可能改变无机化合物的吸湿性 ${ }^{[26]}$. 有 机物质表现核化活性的模式也各有不同, 如顺丁烯 二酸在凝华核化模式中作为冰核 ${ }^{[27]}$, 乙二酸二水合 物则在浸润冻结模式中产生异质核化 ${ }^{[28]}$. Diehl 等 ${ }^{[29]}$ 已经发现许多花粉在湿空气 $(95 \% \mathrm{RH})$ 中可以吸收的 水分达到它们重量的 $100 \%$, 认为这可能与其组成物 质的化学基团化学键的空间物理结构有关 ${ }^{[30]}$.

而本研究所用自来水分别取自中国科学院研究 生院( $\mathrm{G}$ 自来水)和中国国家气象局实验室( $\mathrm{W}$ 自来水), 自来水液滴的平均冻结温度分别是 $(-19.9 \pm 2.7)$ 和 $(-17.1 \pm 3.0)^{\circ} \mathrm{C}$, 稍高于超纯水的平均冻结温度, 但低 于 $\mathrm{Du}$ 和 Ariya ${ }^{[23]}$ 对加拿大 $\mathrm{McGill}$ 大学自来水的测定 结果 $(-11.2 \pm 2.4)^{\circ} \mathrm{C}$, 这可能与自来水中杂质成分及其 含量不同有关. Pruppacher 和 Neiburger ${ }^{[31]}$ 测试可溶性
盐对冰核化温度的影响时发现: 溶质含量增加, 核化 温度在低盐浓度时首先增加, 这是因为伴随溶质最 初的不溶物作为冰核的缘故; 然后高盐浓度时核化 温度降低, 可能是因为冻结点随数浓度变化其核化 特性降低, 降低的程度可能大于增加的有效冰核的 效应. 本研究的不同溶液浓度与冻结温度的变化未 发现类似的变化趋势, 具体原因有可能是液滴冻结 法便于浸润冻结核模式的核化过程的测试. 尽管研 究已经表明同种物质颗粒物可以四种模式进行核化, 但由于物化特性的差异, 其成冰的核化模式各有侧 重, 而本研究的受试物质并非是有效浸润核从而未 显示出较强冻结活性.

\section{2 典型大气污染物对冰核细菌和普通细菌冻结 温度的影响}

\subsection{1 污染物 (乙酸钠、乙二酸和硫酸铵)的独立作 用效应}

已有研究数据表明多种溶质的共存可降低冰核 核化温度, 甚至比均衡熔点降低还多 ${ }^{[32]}$. 本研究以 $\mathrm{PS}^{0}$ 为大气中普遍存在的非冰核细菌的代表, 模拟研 究典型大气污染物(MCA、DCA、无机盐的代表物) 乙酸钠、乙二酸和硫酸铵在大气环境中与之相遇后, 其对于云水液滴冻结温度可能产生的影响. 结果如 图 2 所示: 3 种不同污染物质的添加, 不论是冰核细 菌 PS 菌液还是普通细菌 $\mathrm{PS}^{0}$ 菌液, 在不同浓度 $\left(10^{6}\right.$ 和 $10^{4}$ cells $\mathrm{mL}^{-1}$ ) 其液滴的冻结温度均未发现污染物 有促进冰核核化的现象, 反而在不同程度上降低了 菌液原有的冻结温度, 这种现象的出现可能是溶质 效应导致的, Du 和 Ariya ${ }^{[23]}$ 的测试实验也发现了多种

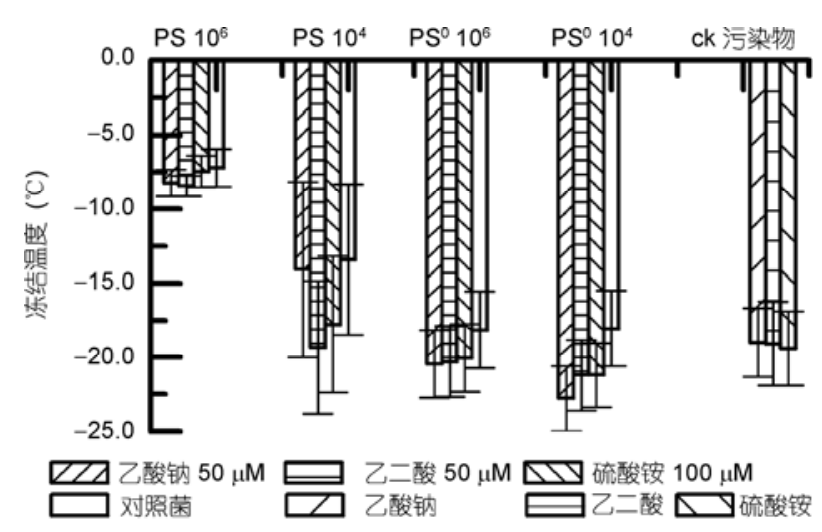

图 2 单种污染物对不同细菌 PS/PS ${ }^{0}$ 液滴冻结温度的影响 
DCA 溶质的复合反而降低了等浓度单种溶液液滴的 冻结温度. 但反过来, 在相同浓度条件下, 当这些污 染物溶液与 $\mathrm{PS}^{0}$ 相遇时, 其液滴的冻结温度不论细菌 数量浓度的多少 $\left(10^{6}\right.$ 和 $10^{4}$ cells $\left.\mathrm{mL}^{-1}\right)$, 混合溶液液 滴的冻结温度同样也比同等浓度不添加菌液的污染 物平均冻结温度低, 似乎表明大气中细菌气溶胶浓 度的增加对于污染物溶液液滴的冻结并没有促进; 然而当与 PS 相遇, 低浓度 $10^{4}$ cells $\mathrm{mL}^{-1} \mathrm{PS}$ 液滴的平 均冻结温度 $\left((-13.4 \pm 5.1)^{\circ} \mathrm{C}\right)$, 比不添加菌液的单种污 染物液滴的平均冻结温度高, 相对于受试污染物溶 液液滴的冻结温度表现出了中等程度的有效冰核活 性, 并促进了乙酸钠 $\left((-19.0 \pm 2.3)^{\circ} \mathrm{C}\right)$ 的有效冰核活性, 而当 PS 浓度增加时 $\left(10^{6}\right.$ cells $\left.\mathrm{mL}^{-1}\right)$, 污染物与菌混合 液的冻结温度都显示了出明显的有效冰核活性, 不 论有机污染物 (MCA 和 DCA) 还是无机污染物其溶液 液滴平均冻结温度都在 $-10^{\circ} \mathrm{C}$ 以上. 尽管污染物降低 了菌液冻结核活性, 本实验结果还是显示出冰核细 菌 PS 即便在浓度较低时也可以通过某些方式而促进 其他物质的冰核活性的, 而非冰核活性细菌则未显 示出此种特性.

大气中有机污染物如 MCA 和 DCA 可以作为细 菌和真菌的营养物, 通过改变有效冰核的整体数量 从而产生冰核活性. Amato 等 ${ }^{[33]}$ 发现大气中的低温细 菌含有转化单元羧酸类化合物 (甲酸盐、乙酸盐和乳 酸盐) 以及甲醛和甲醇的酶系. 他们从云中分离培养 到 128 株微生物, 其中有 71 株是细菌菌种; 在所测试 的 20 株细菌中有 11 株 (55\%)是可以在接近自然大气 环境(i.e. $5^{\circ} \mathrm{C}$ )中生长代谢 ${ }^{[34]}$. 研究表明通常云水中有 机物降解时间周期是几天到几十天, 例如 Herlihy 等 ${ }^{[35]}$ 测得 4 9 月的雨水样品中细菌浓度是 $10^{5}$ cells $\mathrm{mL}^{-1}$, 甲酸盐和乙酸盐的浓度分别是 6 23 和 3 9 $\mu \mathrm{mol} \mathrm{L}{ }^{-1}$; 室温培养至少三天后, 两者的转化速率常 数与转化周期分别是 $0.14 \mathrm{~h}^{-1}, 0.17 \mathrm{~h}^{-1}$ 和 $1.5 \mathrm{~d}, 34 \mathrm{~d}$. Vaïtilingom 等 ${ }^{[36]}$ 模拟云水中分离的微生物在云水溶 液微环境中, 发现 $5^{\circ} \mathrm{C}$ 降解甲酸盐、乙酸盐和丁二酸 盐的生命周期分别是 2.0, 69.1 和 $1.5 \mathrm{~d}$. Bakermans 和 Mark ${ }^{[3]]}$ 研究表明微生物在 $-5 \sim-32^{\circ} \mathrm{C}$ 温度范围的冰相 中只有代谢活动但并不进行生长和繁殖, 归其原因 是受冰的固相物理结构所限. 本研究中大气污染物 溶液与菌液的混合液样品是即配即测, 既使因为测 样时间的意外拖延, 其在 $4^{\circ} \mathrm{C}$ 暂存从未超过 $24 \mathrm{~h}$, 因 此本实验待测溶液, 基本不存在污染物降解或 IN 增
殖的变化, 污染物与菌液的混合仍表现其各自的冰 核活性.

\subsection{2 污染物间的混合效应}

本研究假设混合溶液中污染物质乙酸钠、乙二酸 和硫酸铵之间不产生化学反应, 配制细菌数浓度依 次从 $10^{6}$ cells $\mathrm{mL}^{-1}$ 降低到 $10^{3}$ cells $\mathrm{mL}^{-1}$ 的菌液, 向 菌液中添加三种污染物配制的复合溶液, 使混合液 中三种溶质终浓度依次分别为 $\mathrm{P} 1\left(100 \mu \mathrm{mol} \mathrm{L} \mathrm{L}^{-1}: 100\right.$ $\left.\mu \mathrm{mol} \mathrm{L} \mathrm{L}^{-1}: 200 \mu \mathrm{mol} \mathrm{L}-1\right), \mathrm{P} 2\left(50 \mu \mathrm{mol} \mathrm{L}^{-1}: 50 \mu \mathrm{mol} \mathrm{L}^{-1}\right.$ : $\left.100 \mu \mathrm{mol} \mathrm{L}{ }^{-1}\right)$ 和 P3 $\left(25 \mu \mathrm{mol} \mathrm{L}^{-1}: 25 \mu \mathrm{mol} \mathrm{L}^{-1}: 50 \mu \mathrm{mol}\right.$ $\mathrm{L}^{-1}$ ), 从而模拟大气中云滴的冻结过程. 图 3 是大气 主要污染物对普通细菌 $\mathrm{PS}^{0}$ 和冰核活性细菌 PS 的浸 润核冻结的影响作用的结果. 测试结果显示: 在低菌 浓度级数 $\left(10^{3}\right.$ cells $\left.\mathrm{mL}^{-1}\right)$ 时, 与 $\mathrm{P} 1, \mathrm{P} 2, \mathrm{P} 3$ 混合的冰核 细菌 PS 和普通细菌 $\mathrm{PS}^{0}$ 溶液液滴冻结活性没有明显 差异, 都低于 $-17^{\circ} \mathrm{C}$, 污染物并未显著改变菌液的冻 结活性. 不论是普通细菌还是冰核活性细菌, 当与较 高浓度的污染物复合液 P1 和 P2 混合后, 其冰核活性 也并没有增强, 而且在相同菌数浓度下, P1 和 P2 的 添加反而不同程度地降低了菌液的平均冻结温度. 但低浓度的 P3 溶液(接近当前污染大气水平)却能提 升较高浓度 $\left(10^{6}\right.$ cells $\left.\mathrm{mL}^{-1}\right) \mathrm{PS}^{0}$ 的液滴的冰核活性; 而 P3 对于所有浓度 PS 的液滴冻结温度都有不同程 度的提高. 这个实验结果显示: 在当前的污染大气条 件下, 即便大气中仅有少量的冰核活性细菌 PS 的存 在, 也可能会有效地促进其他冰核的异质核化过程 进而促进降水的形成, 大气细菌气溶胶确实存在着 改变气候变化的可能性.

\section{3 混合污染物溶液中冰核细菌与普通细菌之间 的相互作用}

为进一步模拟真实大气中的冰核浸润核化过程, 基于 Santl-Temkiv 等 ${ }^{[38]}$ 观测发现雨水样品中仅有 $9 \%$ 的细菌可作为有效冰核而大部分的细菌并不具有冰 核活性的实验现象, 我们将大气冰核细菌与普通细 菌的混合情况简化配制了 1.3.3 所述的 A1, A2, A3, B1, B2, B3, C1, C2, C3 菌混合液, 计数结果显示, 混 合液中 PS 菌数占总菌数比分别为 $33 \%, 20 \%$ 和 $11 \%$, 冻结温度测试结果如图 4 显示: $10^{6}$ cells $\mathrm{mL}^{-1}$ 时, 混合液中 PS 比例增减并不影响其冻结温度, 但明 显改变了 $\mathrm{PS}^{0}$ 和污染物的冻结活性, 使污染条件下 

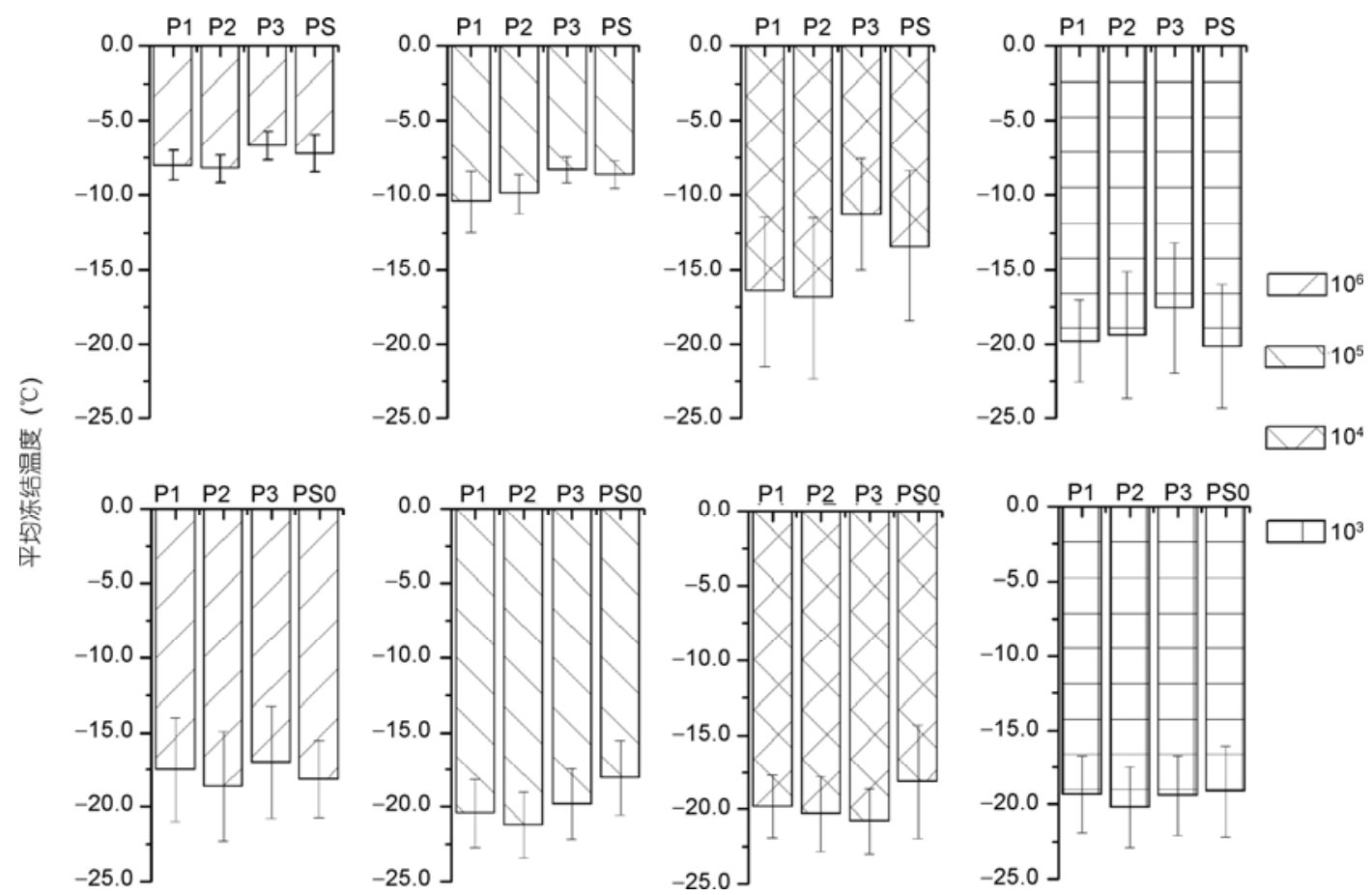

图 3 复合污染物与冰核细菌 $\mathrm{PS} /$ 普通细菌 $\mathbf{P S}^{\mathbf{0}}$ 的相互作用

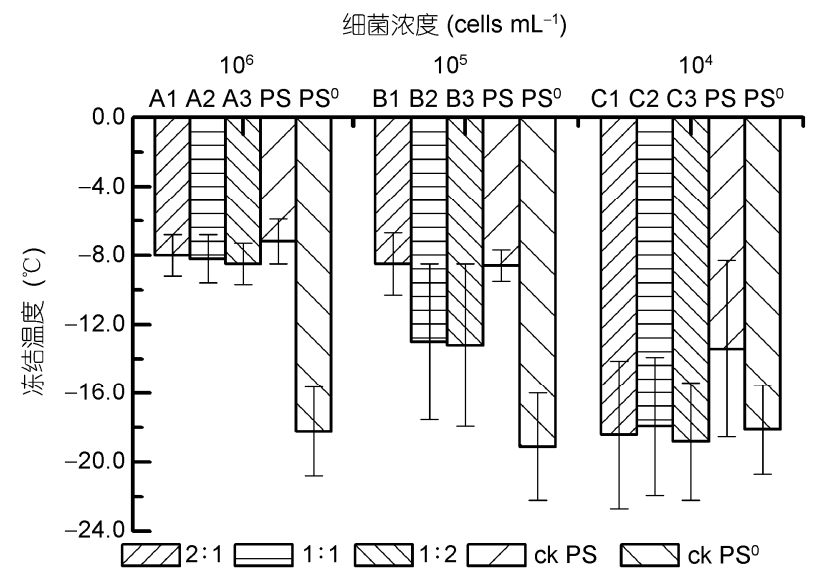

图 4 冰核活性细菌 PS 与普通细菌 PS $^{0}$ 对混合溶液液滴冻 结温度的影响

$\mathrm{PS}^{0}$ 混合液冻结温度接近 PS 的冻结温度; 冰核的累 积核谱也显示, $10^{6}$ cells $\mathrm{mL}^{-1}$ 时, 3 种不同比例浓度的 菌液有效累积核的谱线变化一致, 有效冰核数量都 集中在较高温度范围内, 容易启动冰核的核化过程, 因此可以在较高温度下实现冻结. 同样, 当菌浓度级 数是 $10^{5}$ cells $\mathrm{mL}^{-1}$ 时仅当冰核活性细菌浓度比例较 高(B1)时, 混合液才表现出相同的结果, 但是当冰核 细菌数量比例降低至混合液 B2, B3 时, 虽然有效累积

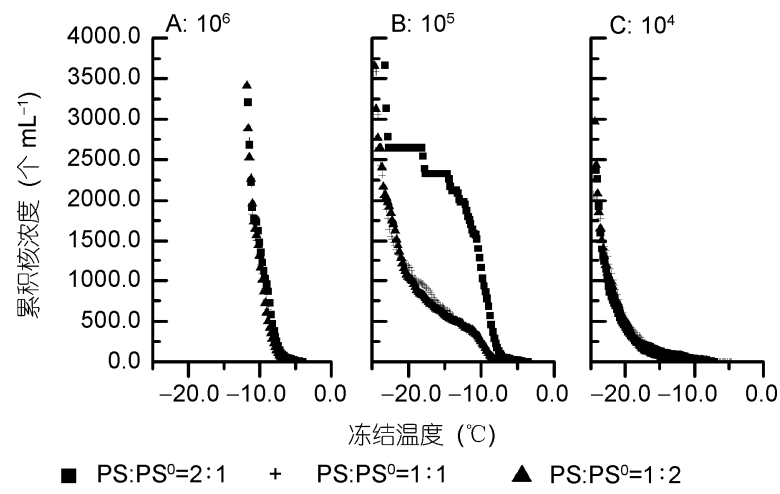

图 5 复合污染物与细菌混合液液滴冰核累积核谱分布

核的温度分布区间向低温区偏移(图 5), 但还是促进了 普通细菌与污染物混合液的冻结活性. 但当冰核细菌 浓度级数较低 $10^{4}$ cells $\mathrm{mL}^{-1}$ 时, 其未能显示出对于 混合液液滴的冻结活性的影响. 说明在液滴冷台测试 条件下, 污染较严重的大气中 PS 浓度数量级是液滴 浸润冻结核化活性的重要影响因素, $10^{6}$ cells $\mathrm{mL}^{-1}$ 的 浓度值是保证其能够发挥高效冰核活性的浓度阈值.

\section{3 结论}

(1) 典型大气污染物(单元羧酸和二元羧酸以及 
无机盐硫酸铵) 在液滴的浸润冻结核化过程中, 并未 显示有效冰核特性, 作为添加的溶质没有升高超纯 水液滴的冻结温度, 而且其溶液浓度的改变对液滴 冻结温度的影响也没有显示出规律性.

(2) 细菌气溶胶分别与典型大气污染物(乙酸钠、 乙二酸和硫酸铵)单独作用时, 这些污染物质不同程 度降低了冰核细菌 PS 和普通细菌 $\mathrm{PS}^{0}$ 菌液的冻结温 度, 减弱了冰核细菌的高效冰核活性; 而冰核细菌 PS 即使较低浓度下也可促进典型大气污染物乙酸钠 的冻结核活性, 当浓度增至 $10^{6}$ cells $\mathrm{mL}^{-1}$ 时, 则能显 著改变乙酸钠、乙二酸和硫酸铵的冻结核活性, 但未 发现普通细菌 $\mathrm{PS}^{0}$ 有此特性.

(3) 模拟大气复合污染环境发现, 复合污染物 溶液与较低的菌浓度级数菌液混合后, 不论冰核菌 还是普通菌与复合污染物之间的相互作用对溶液液 滴的冻结温度没有显著影响. 当所模拟的复合污染 物溶液接近或略高于当前污染大气水平时, 不仅促 进了冰核细菌 PS 的冻结核活性, 提高了其液滴的冻 结温度, 而且对于较高浓度级数 $10^{6}$ cells $\mathrm{mL}^{-1}$ 的普 通菌 $\mathrm{PS}^{0}$ 的冻结核活性也显示出了促进效应. 表明在 当前大气环境条件下, 细菌气溶胶确实有可能通过 改变气溶胶的冻结核化活性而影响降水过程和气候 变化.

(4) 模拟研究显示随着大气环境污染的加剧, 冰 核活性细菌的浓度值是重要的影响因素, 其高效冰 核活性的菌浓度阈值级数是 $10^{6}$ cells $\mathrm{mL}^{-1}$; 此研究 结果对于人工影响天气抗旱增雨过程中, 人工冰核
的选择和优化具有一定科学意义.

当前的研究已经发现真实大气环境中微生物参 与了大气有机污染物的降解过程, 其降解周期达几 天或几十天, 在本研究的测试实验中, 多数测试样品 是即配即测, 既便因意外而拖延了测样时间, 样品在 $4^{\circ} \mathrm{C}$ 暂存从未超过 $24 \mathrm{~h}$, 因此本模拟测试实验与实际 大气成云过程有较大偏差; 而且测试的液滴大小也 远远大于实际大气云中冰核的粒径尺度; 此外在计 算过程中, 我们假定液滴从开始冻结时一直保持其 球形结构, 同时也忽略溶质是否混合充分与均匀. 由 于溶解度低的溶质, 在配置溶液时会由于存在尚未 完全溶解的溶质所形成的初始冰核胚胎, 而提前形 成了液滴的异质冰核结构, 类似问题在我们的实验 中也统统被简化或忽略了. 此外由于本文采用的液 滴冷台实验技术, 局限于浸润核单一冻结模式的测 试, 与云环境条件下多种核化模式交互发生的实际 状况有一定的区别, 模拟实验结果在一定程度上反 映了冰核细菌及不同污染组分混合后的浸润冻结模 式的成冰性能, 为进一步揭示云中细菌冰核核化理 论机制奠定了基础. 而利用液滴的冻结核数量浓度 $\left(\right.$ 个 $\left.\mathrm{mL}^{-1}\right)$, 简化了不同粒径尺度的差异, 可以在一定 程度上反映核化活性. 但在今后的科学研究中, 应该 更充分地考虑待测物质的物理化学和生物特性的变 化, 诸如微生物的代谢过程对溶质的化学成分的变 化、可溶性、表面活性、亲水性以及形态变化等因素 的效应, 从而为更有效地模拟真实大气条件下可能 发生的核化过程.

作者真诚地感谢中国气象科学研究院杨绍忠研究员在整个实验期间所给予的热心帮助和建议; 同时也衷心 地感谢中国科学院微生物研究所周宇光研究员和刘英吴高级实验师所给予的帮助与指导, 最后对审稿专家 给予本文的建议和指导表示诚挚的谢意.

\section{参考文献}

1 DeMott P J, Prenni A J, Liu X, et al. Predicting global atmospheric ice nuclei distributions and their impacts on climate. Proc Natl Acad Sci USA, 2010, 107: 11217-22

2 Bauer H, Giebl H, Hitzenberger R, et al. Airborne bacteria as cloud condensation nuclei. J Geophys Res, 2003, 108: AAC2/1-AAC2/5

3 Franc G D, Demott P J. Cloud activation of airborne Erwinia carotovora cells. J Appl Meteorol, 1998, 37: 1293-1300

4 Morris C E, Georgakopoulos D G, Sands D C. Ice nucleation active bacteria and their potential role in precipitation. J Phys IV France, 2004, 121: $87-103$

5 Kanakidou M, Seinfeld J H, Pandis S N, et al. Organic aerosol and global climate modelling: A review. Atmos Chem Phys, 2005, 5: $1053-1123$

6 Acker K, Mertes S, Moller D, et al. Case study of cloud physical and chemical processes in low clouds at Mt. Brocken. Atmos Res, 2002, 
64: 41-51

7 Marinoni A, Laj P, Sellegri K, et al. Cloud chemistry at the Puy de Dôme: Variability and relationships with environmental factors. Atmos Chem Phys Discuss, 2004, 4: 849-886

8 Sun J, Ariya P A. Atmospheric organic and bio-aerosols as cloud condensation nuclei (CCN): A review. Atmos Environ, 2006, 40, 5: $795-820$

9 Kawamura K, Usukura K. Distributions of low molecular weight dicarboxylic acids in the North Pacific aerosol samples. J Oceanogr, 1993, 49: 271-283

10 Kerminen V M, Ojanen C, Pakkanen T, et al. Low-molecular-weight dicarboxylic acids in an urban and rural atmosphere. J Aerosol Sci, 2000, 3: 349-362

11 Pruppacher H R, Klett J D. Microphysics of Clouds and Precipitation. 2nd ed. Dordrecht: Kluwer Academic Publishers, 1997

12 Ariya P A, Amyot M. The role of bioaerosols in atmospheric chemistry and physics. Atmos Environ, 2004, 38: 1231-1232

13 Ariya P A, Sun J, Eltouny N A, et al. Physical and chemical characterization of bioaerosols-Implications for nucleation processes. Int Rev Phys Chem, 2009, 28: 1-32

14 Maki L R, Willoughby K J. Bacteria as biogenic sources of freezing nuclei. Appl Meteor, 1978, 17: 1049-1053

15 Mortazavi R, Hayes C T, Ariya P A. Ice nucleation activity of bacteria isolated from snow compared with organic and inorganic substrate. Environ.Chem, 2008, 5: 373-381

16 Wang G H, Niu S L, Liu C, et al. Identification of dicarboxylic acids and aldehydes of PM 10 and $\mathrm{PM}_{2.5}$ aerosols in Nanjing, China. Atmos Environ, 2002, 36: 1941-1950

17 Kawamura K, Ikushima K. Seasonal change in the distribution of dicarboxylic acids in the urban atmosphere. Environ Sci Technol, 1993, 27: 2227-2235

18 Sempere R, Kawamura K. Comparative distributions of dicarboxylic acids and related polar compounds in snow, rain and aerosols from urban atmosphere. Atmos Environ, 1994, 28: 449-459

19 胡敏, 张静, 吴志军. 北京降水化学组成特征及其对大气颗粒物的去除作用. 中国科学 B 辑: 化学, 2005, 35: 169-176

20 Delort A M, Vaïtilingom M, Amato P, et al. A short overview of the microbial population in clouds: Potential roles in atmospheric chemistry and nucleation processes. Atmos Res, 2010, 98: 249-260

21 杨绍忠, 豐大雄. 一个检测水中冻结核含量的新装置. 气象学报, 2007, 65: 976-982

22 Vali G. Quantitative evaluation of experimental results on the heterogeneous freezing nucleation of supercooled liquids. Atmos Sci, 1971, 28: 402-409

23 Du R, Ariya P A. The freezing temperature of C2-C6 dicarboxylic acid: The important indicator for ice nucleation processes. Chin Sci Bull, 2008, 53: 2685-2691

24 Fukuta N. Experimental studies of organic ice nuclei. Atmos Sci, 1966, 23: 191-196

25 Gavish M, Wang J L, Eisenstein M, et al. The role of crystal polarity in alpha-amino acid crystals for induced nucleation of ice. Science, 1992, 256: 815-818

26 Wise M E, Garland R M, Tolbert M A. Ice nucleation in internally mixed ammonium sulfate/dicarboxylic acid particles. J Geophys Res, 2004, 109: D19203, doi: 10.1029/2003JD004313

27 Shilling J E, Fortin T J, Tolbert M A, et al. Depositional ice nucleation on crystalline organic and inorganic solids. J Geophys Res, 2006, 111: D12204

28 Zobrist B, Marcolli C, Koop T, et al. Oxalic acid as a heterogeneous ice nucleus in the upper troposphere and its indirect aerosol effect. Atmos Chem Phys, 2006, 6: 3115-3129

29 Diehl K, Quick C, Matthias-Maser S, et al. The ice nucleating ability of pollen. Part I: Laboratory studies in deposition and condensation freezing modes. Atmos Res, 2001, 8: 75-87

30 Paul S, Hazra A, De U K, et al. Comparative study of nucleation by different alcoholic solutions of benzoin, and benzoin dust. J Atmos Chem, 2006, 53: 155-168

31 Pruppacher H R, Neiburger M. The effect of water-soluble substances on the supercooling of water drops. Atmos Sci, 1963, 20: 376-385

32 Wilson P W, Heneghan A F, Haymet A D J. Ice nucleation in nature: Supercooling point (SCP) measurements and the role of heterogeneous nucleation. Cryobiology, 2003, 46: 88-98

33 Amato P, Ménager M, Sancelme M, et al. Microbial population in cloud water at the Puy de Dôme: Implications for the chemistry of clouds. Atmos Environ, 2005, 39: 4143-4153

34 Amato P, Parazols M, Sancelme M, et al. Microorganisms isolated from the water phase of tropospheric clouds at the Puy de Dôme: Major 
groups and growth abilities at low temperatures. FEMS Microbiol Ecol, 2007, 59: 242-254

35 Herlihy L J, Galloway J N, Mills A L. Bacterial utilization of formic and acetic acid in the rainwater. Atmos Environ, 1987, 21: 23972402

36 Vattilingom M, Charbouillot T, Deguillaume L, et al. Atmospheric chemistry of carboxylic acids: Microbial implication versus photochemistry. Atmos Chem Phys, 2011, 11: 8721-8733

37 Bakermans C, Mark L. Microbial metabolism in ice and brine at $-5^{\circ} \mathrm{C}$. Environ Microbiol, 2011, 13: 2269-2278

38 Santl-Temkiv T, Gosewinkel-Karlson U, Finster K, et al. The diversity and proportion of ice nucleation active bacteria in rain and their ability to produce extracellular ice nucleation active particles. In: Smolík J, O’Dowd C, eds. 18th International Conference on Nucleation and Atmospheric Aerosols (ICNAA). Prague, Czech Republic, 2009. 1460-1466 